\title{
Schätzung genetischer Parameter für Testtagsergebnisse von Milchleistungsmerkmalen bei Bunten Deutschen Edelziegen
}

\begin{abstract}
Title of the paper: Estimation of genetic parameters for test day records of milk performance traits in German Improved Fawn

The objectives of this study were to estimate genetic parameters for milk performance traits of German Improved Fawn by using univariate und multivariate animal models. The analysis was based on 27,778 test day records of 1,848 German Improved Fawn with 3,574 lactation records distributed over 229 flocks in Lower Saxony, Saxony and Baden-Wuerttemberg. Milk records were sampled between 1988 and 2002. The animals in our analysis were the progeny of 455 sires and 1.148 does. Heritabilities estimated with a multivariate test day model with fixed regression were $\mathrm{h}^{2}=0.19,0.16$ and 0.15 for milk, fat and protein yield. For fat and protein content and Somatic Cell Score (SCS) heritabilities were $\mathrm{h}^{2}=0.17,0.14$ and 0.16 , respectively. The additive genetic correlations between milk yield and fat as well as protein yield of German Improved Fawn were very high and positive $\left(r_{g}=0.84\right.$ and $\left.r_{g}=0.77\right)$. Milk yield and milk contents were genetically negative correlated with $r_{g}=-0.28$ for fat and $r_{g}=-0.22$ for protein content. A moderate additive genetic correlation $\left(r_{g}=0.48\right)$ between fat and protein content was estimated. There were no considerable additive genetic correlations between fat yield and protein content as well as between fat content and protein yield $\left(r_{g}=0.05\right.$ and $\left.r_{g}=0.09\right)$. Additive genetic correlations between milk, fat or protein yield and SCS were high and negative, whereas additive genetic correlations between fat or protein content and SCS were low and positive. The genetic parameters estimated from field test records allow to achieve genetic progress in milk performance traits of German Improved Fawn.
\end{abstract}

Key Words: goats, milk performance traits, genetic parameters

\section{Zusammenfassung}

Ziel dieser Arbeit war die Analyse von Milchleistungsmerkmalen Bunter Deutscher Edelziegen mit univariaten und multivariaten Testtagstiermodellen. Dazu standen 27.778 Testtagsergebnisse aus 3.574 Laktationen von 1.848 Bunten Deutschen Edelziegen zu Verfügung. Die Tiere wurden in 229 Betrieben im Bereich des Ziegenzuchtverbandes Niedersachsen, des Sächsischen Schaf- und Ziegenzuchtverbandes und des Ziegenzuchtverbandes Baden-Württemberg gehalten. Ausgewertet wurden Milchleistungsdaten aus den Jahren 1988 bis 2002. Die milchleistungsgeprüften Tiere stammten von 455 Vätern und 1.148 Müttern ab. Mit einem multivariaten Testtagstiermodell mit fixer Regression wurden für die Milch-, Fett- und Eiweißmenge Heritabilitäten von $\mathrm{h}^{2}=0,19$, 0,16 und 0,15 sowie für den Fett- und Eiweißgehalt und den Somatic Cell Score (SCS) Heritabilitäten von $\mathrm{h}^{2}=$ 0,17, 0,14 und 0,16 geschätzt. Hohe additiv-genetische Korrelationen wurden zwischen der Milchmenge und der Fett- bzw. Eiweißmenge ermittelt ( $r_{\mathrm{g}}=0,84$ bzw. $\left.\mathrm{r}_{\mathrm{g}}=0,77\right)$, während die Milchmenge zum Fett- und Eiweißgehalt genetisch negativ $\left(r_{g}=-0,28\right.$ bzw. $\left.r_{g}=-0,22\right)$ korreliert war. Zwischen dem Fett- und Eiweißgehalt bestand eine mittlere positive additiv-genetische Korrelation $\left(r_{\mathrm{g}}=0,48\right)$. Niedrige additiv-genetische Korrelationen bestanden hingegen zwischen der Fettmenge und dem Eiweißgehalt sowie zwischen der Eiweißmenge und dem Fettgehalt $\left(r_{\mathrm{g}}=0,05\right.$ bzw. $\left.r_{\mathrm{g}}=0,09\right)$. Die additiv-genetische Korrelationen zwischen dem SCS und der Milch-, Fett- und Eiweißmenge waren hoch und negativ. Im Gegensatz dazu bestanden zwischen dem SCS und dem Fett- und Eiweißgehalt niedrige und positive additiv-genetische Korrelationen. Die gefundenen genetischen Parameter erlauben mittels Selektionsprogrammen einen Zuchtfortschritt für Milchleistungsmerkmale.

Schlüsselwörter: Milchziegen, Milchleistungsmerkmale, genetische Parameter

1. Einleitung

In Deutschland ist die Bunte Deutsche Edelziege am weitesten verbreitet. Sie zeichnet sich durch eine hohe Milchleistung und Fruchtbarkeit sowie eine lange Nutzungsdauer 
aus. Spitzenleistungen von bis zu $1900 \mathrm{~kg}$ Milch pro Laktation sind möglich. Die Bunte Deutsche Edelziege stellt etwa 65\% des Milchziegenbestandes in Deutschland dar.

Sie ging Ende des 19. und zu Beginn des 20. Jahrhunderts durch Selektion aus den verschiedenen bunten Landschlägen in Deutschland hervor. Zwar wurden zur Verbesserung der bunten Ziegen auch einige Ziegen aus der Schweiz eingekreuzt, insgesamt spielte die Einkreuzung bei dieser Rasse jedoch nur eine geringe Rolle (GALL, 2001). Im Jahre 1928 wurden alle farbigen Ziegenschläge unter der Bezeichnung „Bunte Deutsche Edelziege“ zusammengefasst, um eine größere Zuchtbasis zu erhalten.

Trotz der zunehmenden Bedeutung dieser Ziegenrasse als Milchproduzent wurde das Leistungspotenzial der Tiere in den letzten Jahrzehnten kaum durch tierzüchterische Maßnahmen verbessert, und es liegen keine Untersuchungen zu den genetischen $\mathrm{Pa}$ rametern der Milchleistungsmerkmale vor. Deren Kenntnis ist aber Voraussetzung für die Durchführung von Zuchtprogrammen.

Ziel dieser Arbeit ist es deshalb, die genetischen Parameter der Milchleistung der Bunten Deutschen Edelziegen zu analysieren. Es sollen Heritabilitäten und genetische und residuale Varianz-Kovarianzmatrizen für die Milch-, Fett- und Eiweißmenge, den Fett- und Eiweißgehalt sowie den somatischen Zellgehalt in einem Testtagsmodell mit fixer Regression geschätzt werden.

\section{$2 . \quad$ Material und Methode}

Das aus der Milchleistungsprüfung vorliegende Datenmaterial wurde vom VIT Verden, dem Landeskontrollverband Sachsen und der Rinderunion Baden-Württemberg zur Verfügung gestellt. Insgesamt wurden 27.778 Probemelkergebnisse aus 3.574 Laktationen von 1.848 Bunten Deutschen Edelziegen berücksichtigt, deren Pedigreedaten zur Verfügung standen. Weitere Materialbeschreibungen sind bei BÖMKES et al. (2004a) zu finden.

Die milchleistungsgeprüften Bunten Deutschen Edelziegen waren die Töchter von 455 Vätern und 1.148 Müttern, die wiederum von 274 Großvätern und 336 Großmüttern väterlicherseits sowie 401 Großvätern und 733 Großmüttern mütterlicherseits abstammten. Durchschnittlich 3,9 Probanden stammten von einem Bock ab. Während die größte Gruppe väterlicher Halbgeschwister aus 38 Tieren bestand, war von 38,9\% der Böcke nur jeweils eine Tochter in der Milchleistungsprüfung. Außerdem bestand das Probandenmaterial aus 216 Vollgeschwisterfamilien, von denen 178 jeweils zwei Töchter, 36 jeweils drei Töchter und zwei jeweils vier Töchter umfassten.

Von den 4.479 Tieren in der Pedigreedatei waren 1.169 Gründertiere, also Tiere ohne bekannte Eltern, und 3.310 Tiere mit bekannten Eltern.

Die durchschnittliche Laktation der Bunten Deutschen Edelziegen, gemessen vom Ablammdatum bis zum letzten Probemelktermin, dauerte 234,1 \pm 76,4 Tage. Während dieser Zeit produzierten die Tiere im Durchschnitt pro Tag 2,87 \pm 1,20 kg Milch mit einem Fettgehalt von 3,08 \pm 0,54\% und einem Eiweißgehalt von 3,38 \pm 0,95\%. Die somatische Zellzahl wurde für die Auswertung zum Somatic Cell Score (SCS) transformiert. Der SCS betrug im Durchschnitt 5,49 \pm 1,93. Die Rohmittelwerte der täglichen Milchleistung sind der Tabelle 1 zu entnehmen. Während des Zeitraums der Milchleistungsprüfung wurde eine durchschnittliche Gesamtleistung pro Laktation von $671,9 \pm 280,9$ kg Milch mit 20,7 \pm 8,7 kg Fett und 22,7 \pm 9,5 kg Eiweiß erbracht. 
Tabelle 1

Anzahl Testtagsergebnisse (n), Mittelwerte $(\bar{x})$, Standardabweichungen (s), Minima (Min) und Maxima (Max) der Testtagsergebnisse der Milchleistungsmerkmale der Bunten Deutschen Edelziegen (Number of test day records (n), means $(\bar{x})$, standard deviations (s), minima (Min) and maxima (Max) of daily milk production traits of German Improved Fawn)

\begin{tabular}{lccccc}
\hline Merkmal & $\mathrm{n}$ & $\bar{x}$ & $\mathrm{~s}$ & Min & Max \\
\hline Milch-kg & 38011 & 2,87 & 1,20 & 0,1 & 10,2 \\
Fett-g & 37701 & 86,7 & 36,3 & 3,3 & 511,6 \\
Eiweiß-g & 37724 & 95,4 & 47,6 & 2,6 & 724,1 \\
Fett-\% & 37711 & 3,08 & 0,54 & 0,94 & 9,99 \\
Eiweiß-\% & 37734 & 3,38 & 0,95 & 0,69 & 9,99 \\
SCS & 4449 & 5,49 & 1,93 & $-3,64$ & 11,18 \\
\hline
\end{tabular}

Für die einzelnen Milchleistungsmerkmale Milch-, Fett- und Eiweißmenge, Fett- und Eiweißgehalt und SCS wurde zunächst eine univariate Varianzkomponentenschätzung mittels eines Testtagstiermodells mit fixer Regression durchgeführt. Anschließend erfolgte zur Schätzung der additiv-genetischen und residualen Varianz-Kovarianzmatrizen eine multivariate Auswertung. Die Schätzung der Varianzkomponenten erfolgte mit VCE4, Version 4.2.5 (GROENEVELD, 1998). Die additiv-genetischen und residualen Varianzen und Kovarianzen für die Milchleistungsmerkmale der Bunten Deutschen Edelziegen wurden mittels Residual Maximum Likelihood (REML) geschätzt. Aus den geschätzten Dispersionsparametern wurden die Heritabilitäten, die additivgenetischen und residualen Korrelationen sowie deren Standardfehler abgeleitet. Folgende Effekte wurden im Testtagstiermodell mit fixer Regression untersucht:

Uni- und multivariates Modell für die Analyse der Milchleistungsmerkmale der Bunten Deutschen Edelziege

$$
\begin{aligned}
& \mathrm{Y}_{\mathrm{ijklmnopqrs}}=\mu+\mathrm{R}_{\mathrm{i}}+\mathrm{S}_{\mathrm{j}}+\mathrm{J}_{\mathrm{k}}+\mathrm{Z}_{\mathrm{l}}+\mathrm{L}_{\mathrm{m}}+\mathrm{PI}_{\mathrm{n}}+\mathrm{h}(\mathrm{R})_{\mathrm{io}}+\mathrm{b}_{1} * \mathrm{DIM}_{\mathrm{p}}+\mathrm{b}_{2} *\left(\mathrm{DIM}_{\mathrm{p}}\right)^{2}+ \\
& \mathrm{b}_{3} * \log \mathrm{DIM}_{\mathrm{p}}+\mathrm{b}_{4} *\left(\log \mathrm{DIM}_{\mathrm{p}}\right)^{2}+\mathrm{t}_{\mathrm{q}}+\mathrm{a}_{\mathrm{r}}+\mathrm{e}_{\mathrm{ijklmnopqrs}} \\
& \mathrm{Y}_{\mathrm{ijklmnopqr}} \quad=\text { Testtagsergebnis für die Milch-, Fett- und Eiweißmenge, den Fett- und Ei- } \\
& \text { weißgehalt und den SCS des ijklmnopqr-ten Tieres } \\
& \mu \quad=\text { Modellkonstante } \\
& \mathrm{R}_{\mathrm{i}} \quad=\text { fixer Effekt der Region i }(\mathrm{i}=1-3) \\
& \mathrm{S}_{\mathrm{j}} \quad=\text { fixer Effekt des Ablammmonats } \mathrm{j}(\mathrm{j}=1-4) \\
& \mathrm{J}_{\mathrm{k}} \quad=\text { fixer Effekt des Ablammjahres } \mathrm{k}(\mathrm{k}=1-14) \\
& \mathrm{Z}_{\mathrm{l}} \quad \text { = fixer Effekt der Anzahl geborener Lämmer } \mathrm{l}(\mathrm{l}=1-3) \\
& \mathrm{L}_{\mathrm{m}} \quad \text { = fixer Effekt der Laktationsnummer } \mathrm{m}(\mathrm{m}=1-7) \\
& \mathrm{PI}_{\mathrm{n}} \quad \text { = fixer Effekt des Probemelkintervalls } \mathrm{n}(\mathrm{n}=1-170) \\
& \mathrm{h}(\mathrm{R})_{\mathrm{io}} \quad \text { = zufälliger Effekt des Betriebes o innerhalb der Region i }(\mathrm{o}=1-229) \\
& \mathrm{t}_{\mathrm{q}} \quad=\text { zufälliger permanenter Umwelteffekt des Tieres }(\mathrm{q}=1-1.848) \\
& \mathrm{a}_{\mathrm{r}} \quad \text { = zufälliger additiv-genetischer Effekt des Tieres }(\mathrm{r}=1-4.479) \\
& \mathrm{DIM}_{\mathrm{q}} \quad=\text { Tage in Milch als lineare und nichtlineare Kovariable } \\
& \mathrm{b}_{1}-\mathrm{b}_{4} \quad=\text { Regressionskoeffizienten } \\
& \mathrm{e}_{\mathrm{ijklmnopr}} \quad=\text { zufälliger Restfehler }
\end{aligned}
$$

Die Analyse der fixen Effekte wird bei BÖMKES et al. (2004a) beschrieben.

Die genetischen Parameter Heritabilität $\left(\mathrm{h}^{2}\right)$ und Wiederholbarkeit $(w)$ für die Testtagsergebnisse wurden wie folgt geschätzt:

$$
\mathrm{h}^{2}=\sigma_{\mathrm{a}}^{2} /\left(\sigma_{\mathrm{a}}^{2}+\sigma_{\text {tier }}^{2}+\sigma_{\mathrm{e}}^{2}\right) \quad \mathrm{w}=\sigma_{\text {tier }}^{2} /\left(\sigma_{\mathrm{a}}^{2}+\sigma_{\text {tier }}^{2}+\sigma_{\mathrm{e}}^{2}\right) \text {, }
$$

wobei $\sigma_{\mathrm{a}}^{2}$ : additiv-genetische Varianz, 
$\sigma_{\text {tier: }}^{2}$ durch die permanente Umwelt des Tieres bedingte Varianz,

$\sigma^{2} \mathrm{e}$ : Restvarianz.

\section{3. $\quad$ Ergebnisse}

Die Varianzkomponenten zeigten bei uni- und multivariater Auswertung annähernd gleich große Werte (Tab. 2). So betrug für die Milchmenge die additiv-genetische Varianz $\sigma^{2}{ }_{a}=0,136$ bei univariater und $\sigma_{a}^{2}=0,133$ bei multivariater Auswertung. Die additiv-genetische Varianz der Fettmenge nahm mit $\sigma_{a}^{2}=115$ bzw. $\sigma_{a}^{2}=116$ bei unibzw. multivariater Auswertung sehr ähnliche Werte an. Für die Eiweißmenge wurde bei univariater Auswertung eine additiv-genetische Varianz von $\sigma_{a}^{2}=174$ und bei multivariater Auswertung von $\sigma^{2}{ }_{a}=177$ geschätzt. Für den Eiweißgehalt wurden bei beiden Auswertungen deutlich höhere Varianzen geschätzt als für den Fettgehalt. Die additiv-genetische Varianz für den Fettgehalt betrug bei univariater und multivariater Auswertung übereinstimmend jeweils $\sigma_{\mathrm{a}}^{2}=0,045$. Auch für den Eiweißgehalt stimmten die geschätzten additiv-genetischen Varianzen zwischen beiden Auswertungsmethoden mit $\sigma^{2}{ }_{a}=0,083$ überein. Die additiv-genetische Varianz des SCS betrug bei univariater Auswertung $\sigma^{2}{ }_{a}=0,264$ und war bei multivariater Auswertung mit $\sigma^{2}{ }_{a}=$ 0,276 nur geringfügig größer. Im Gegensatz dazu sank die Restvarianz bei multivariater Auswertung mit $\sigma^{2}{ }_{\mathrm{e}}=0,882 \mathrm{im}$ Vergleich zur univariaten Auswertung mit $\sigma_{\mathrm{e}}^{2}=$ 1,871 deutlich ab.

Tabelle 2

Uni- und multivariat mittels REML geschätzte Varianzen der Milchleistungsmerkmale der Bunten Deutschen Edelziegen (Variances of milk yield and milk components of German Improved Fawn estimated by means of univariat and multivariate REML procedures)

\begin{tabular}{lcccccccr}
\hline \multirow{2}{*}{ Merkmal } & \multicolumn{4}{c}{ univariat } & \multicolumn{5}{c}{ multivariat } \\
\cline { 2 - 9 } & $\sigma^{2}{ }_{\mathrm{a}}$ & $\sigma_{\text {tier }}^{2}$ & $\sigma_{\text {betr }}^{2}$ & $\sigma^{2}{ }_{\mathrm{e}}$ & $\sigma^{2}{ }_{\mathrm{a}}$ & $\sigma_{\text {tier }}^{2}$ & $\sigma_{\text {betr }}^{2}$ & $\sigma^{2}{ }_{\mathrm{e}}$ \\
\hline Milch-kg & 0,136 & 0,156 & 0,332 & 0,414 & 0,133 & 0,158 & 0,337 & 0,411 \\
Fett-g & 114,8 & 137,6 & 365,9 & 459,5 & 116,1 & 137,0 & 365,7 & 459,5 \\
Eiweiß-g & 173,6 & 185,9 & 515,8 & 844,6 & 177,2 & 186,7 & 512,0 & 846,7 \\
Fett-\% & 0,045 & 0,008 & 0,022 & 0,113 & 0,045 & 0,008 & 0,022 & 0,210 \\
Eiweiß-\% & 0,083 & 0,056 & 0,060 & 0,377 & 0,083 & 0,058 & 0,066 & 0,433 \\
SCS & 0,264 & 0,537 & 0,875 & 1,871 & 0,276 & 0,596 & 0,787 & 0,882 \\
\hline
\end{tabular}

$\sigma_{\mathrm{a}}^{2}$ : additiv-genetische Varianz, $\sigma_{\mathrm{e}}^{2}$ : Residualvarianz, $\sigma_{\text {tier }}^{2}$ : durch die permanente Tierumwelt bedingte Varianz, $\sigma_{\text {betr }}^{2}$ herdenumweltbedingte Varianz.

Für die durch die permanente Umwelt des Tieres bedingte bzw. für die herdenumweltbedingte Varianz wurden bei univariater und multivariater Auswertung übereinstimmende oder annähernd übereinstimmende Werte geschätzt. Während für die Milchmenge, die Fettmenge, die Eiweißmenge und den SCS die Varianz der permanenten Umwelt des Tieres die additiv-genetische Varianz übertraf, waren für den Fett- und Eiweißgehalt die additiv-genetische Varianzen höher als die Varianzen der permanenten Umwelt des Tieres. Die herdenumweltbedingte Varianz blieb für alle Milchleistungsmerkmale geringer als die Restvarianz.

Die uni- und multivariat geschätzten Heritabilitäten der Milchleistungsmerkmale der Bunten Deutschen Edelziegen variierten zwischen $h^{2}=0,10$ und $h^{2}=0,27$ (Tab. 3). Während für den Fett- und Eiweißgehalt bei der multivariaten Auswertung geringere Werte als bei der univariaten Auswertung geschätzt wurden, stieg der Heritabilitätsschätzwert für die Eiweißmenge und den SCS von der univariaten zur multivariaten Auswertung an. Für die Milchmenge bzw. die Fettmenge wurde bei beiden Auswertungen übereinstimmend eine Heritabilität von $\mathrm{h}^{2}=0,19$ bzw. $\mathrm{h}^{2}=0,16$ geschätzt. Die 
höchste Heritabilität wurde mit $\mathrm{h}^{2}=0,27$ bei univariater Auswertung und auch im Vergleich beider Auswertungen für den Fettgehalt geschätzt. Bei alleiniger Betrachtung der multivariaten Auswertung betrug der höchste Schätzwert $h^{2}=0,19$ für die Milchmenge. Weiterhin lagen die geschätzten Heritabilitäten für die Fettmenge und den Fettgehalt in beiden Auswertungen geringfügig über denen für die Eiweißmenge und den Eiweißgehalt. Die bei der univariaten Auswertung ermittelten Standardfehler lagen zwischen $\mathrm{SE}=0,01$ und 0,03.

Die Wiederholbarkeiten der Milchleistungsmerkmale für die Bunten Deutschen Edelziegen variierten zwischen $\mathrm{w}=0,05$ und $\mathrm{w}=0,34$ (Tab. 3). Zwischen der univariaten und der multivariaten Auswertung stimmten die Wiederholbarkeiten je nach Merkmal annähernd oder genau überein. Die größte Differenz zwischen den beiden Auswertungen bestand für den SCS, dessen Wiederholbarkeit bei univariater Auswertung $\mathrm{w}=$ 0,20 und bei multivariater Auswertung w = 0,34 betrug. Bei der univariaten Auswertung wurde die größte Wiederholbarkeit mit $\mathrm{w}=0,22$ für die Milchmenge ermittelt. Die Standardfehler der Wiederholbarkeit lagen bei univariater Auswertung zwischen $\mathrm{SE}=0,01$ und 0,03 .

Tabelle 3

Uni- und multivariat geschätzte Heritabilitäten $\left(\mathrm{h}^{2}\right)$ und Wiederholbarkeiten (w) der Milchleistungsmerkmale der Bunten Deutschen Edelziegen (Heritability $\left(\mathrm{h}^{2}\right)$ and repeatability $(\mathrm{w})$ estimates of milk production traits and somatic cell score of German Improved Fawn using univariate and multivariate procedures)

\begin{tabular}{lcccc}
\hline \multirow{2}{*}{ Merkmal } & \multicolumn{2}{c}{ univariat } & \multicolumn{2}{c}{ multivariat* $^{*}$} \\
\cline { 2 - 5 } & $\mathrm{h}^{2} \pm \mathrm{SE}$ & $\mathrm{w}$ & $\mathrm{h}^{2}$ & $\mathrm{w}$ \\
\hline Milch-kg & $0,19 \pm 0,02$ & $0,22 \pm 0,01$ & 0,19 & 0,23 \\
Fett-g & $0,16 \pm 0,01$ & $0,19 \pm 0,01$ & 0,16 & 0,19 \\
Eiweiß-g & $0,14 \pm 0,01$ & $0,15 \pm 0,01$ & 0,15 & 0,15 \\
Fett-\% & $0,27 \pm 0,02$ & $0,05 \pm 0,01$ & 0,17 & 0,03 \\
Eiweiß-\% & $0,16 \pm 0,01$ & $0,11 \pm 0,01$ & 0,14 & 0,10 \\
SCS & $0,10 \pm 0,03$ & $0,20 \pm 0,03$ & 0,16 & 0,34 \\
\hline
\end{tabular}

SE: nicht schätzbar.

Tabelle 4

Additiv-genetische (unterhalb der Diagonalen) und residuale (oberhalb der Diagonalen) Korrelationen zwischen den Milchleistungsmerkmalen der Bunten Deutschen Edelziegen (Additive genetic (below diagonal) and residual (above diagonal) correlations between milk production traits of German Improved Fawn)

\begin{tabular}{lcccccc}
\hline Merkmal & Milch-kg & Fett-g & Eiweiß-g & Fett-\% & Eiweiß-\% & SCS \\
\hline Milch-kg & - & 0,87 & 0,37 & $-0,11$ & $-0,02$ & $-0,07$ \\
Fett-g & 0,84 & - & 0,69 & 0,28 & 0,07 & $-0,03$ \\
Eiweiß-g & 0,77 & 0,81 & - & 0,04 & 0,59 & 0,01 \\
Fett-\% & $-0,28$ & 0,28 & 0,09 & - & 0,25 & 0,05 \\
Eiweiß-\% & $-0,22$ & 0,05 & 0,40 & 0,48 & - & 0,48 \\
SCS & $-0,96$ & $-0,66$ & $-0,56$ & 0,12 & 0,17 & \\
\hline
\end{tabular}

Die additiv-genetischen Korrelationen zwischen der Milchmenge und der Fett- und Eiweißmenge waren mit $r_{g}=0,84$ bzw. $r_{g}=0,77$ sehr hoch und positiv (Tab. 4). Ebenfalls positiv und sehr hoch waren mit $r_{e}=0,87$ die residualen Korrelationen zwischen der Milch- und Fettmenge, wohingegen die residualen Korrelationen zwischen der Milch- und Eiweißmenge im mittleren positiven Bereich lagen $\left(r_{e}=0,37\right)$. Im Gegen- 
satz dazu waren die additiv-genetischen Korrelationen zwischen der Milchmenge und dem Fett- und Eiweißgehalt negativ und im mittleren Bereich $\left(r_{g}=-0,28\right.$ bzw. $r_{g}=-$ 0,22). Die residualen Korrelationen zwischen diesen Merkmalen lagen mit $r_{e}=-0,11$ bzw. $r_{e}=-0,02$ im niedrigen und negativen Bereich. Zwischen der Fett- und Eiweißmenge bestanden sehr hohe positive $\left(\mathrm{r}_{\mathrm{g}}=0,81\right)$ und zwischen dem Fett- und Eiweißgehalt hohe positive additiv-genetische Korrelationen $\left(r_{g}=0,48\right)$. Dagegen war der SCS hoch bis sehr hoch, aber negativ mit der Milch-, Fett- und Eiweißmenge additivgenetisch korreliert $\left(r_{g}=-0,56\right.$ bis $\left.-0,96\right)$. Die additiv-genetischen Korrelationen zwischen dem SCS und dem Fett- und Eiweißgehalt lagen im niedrigen, aber positiven Bereich. Die residualen Korrelationen zwischen dem SCS und den Milchleistungsmerkmalen waren negativ oder positiv und sehr gering. Lediglich zwischen dem SCS und dem Eiweißgehalt bestand eine mittlere und positive residuale Korrelation $\left(\mathrm{r}_{\mathrm{e}}=\right.$ $0,48)$.

\section{Diskussion}

In Übereinstimmung mit ANALLA et al. (1996), BAGNICKA und LUKASZEWICZ (1999), MAVROGENIS et al. (1984) und KOMINAKIS et al. (2000) wurde für die Milchmenge der Bunten Deutschen Edelziegen mittels eines Testtagstiermodells mit fixer Regression bei uni- und multivariater Auswertung mittels REML eine Heritabilität von $h^{2}=0,19$ geschätzt. ANALLA et al. (1996) untersuchten Milchleistungsmerkmale von spanischen Murciano-Granadina-Ziegen und ermittelte Heritabilitäten von $h^{2}=0,18$ bei univariater und $h^{2}=0,17$ bei multivariater Auswertung mit einem REML-Tiermodell. Auch BAGNICKA und LUKASZWEWICZ (1999) ermittelten bei ihren Untersuchungen an polnischen Ziegen verschiedener Rassen mit einem Tiermodell mittels REML bei uni- und multivariater Auswertung jeweils einen Heritabilitätsschätzwert von $\mathrm{h}^{2}=0,17$ für die Milchmenge. Bei der Verwendung eines multivariaten Vatermodells erhielten sie allerdings mit $h^{2}=0,48$ einen deutlich höheren Schätzwert. Diese sehr unterschiedlichen Ergebnisse zeigen, dass die Methode der Schätzung und die dabei berücksichtigten genetischen Effekte einen erheblichen Einfluss auf die Schätzergebnisse haben können. Auch RØNNIGEN (1965), ILOEJE et al. (1981), KENNEDY et al. (1982a), MAVROGENIS et al. (1988), BOICHARD et al. (1989), und BÉLICHON et al. (1995) ermittelten mit verschiedenen Auswertungsmethoden (Henderson I, Henderson III, REML-Vater- und Tiermodell) erheblich höhere Schätzwerte für die Milchmenge verschiedener Ziegenrassen. Neben den Auswertungsmethoden können aber auch Unterschiede in der Datenstruktur und der Datenmenge die Schätzergebnisse erheblich beeinflussen. So muss bei einem Vergleich der Ergebnisse beachtet werden, dass die zitierten Autoren die Gesamtlaktationsleistungen von Ziegen verschiedener Rassen untersuchten, wohingegen in dieser Arbeit Testtagsergebnisse ausgewertet wurden. Da bei Testtagsergebnissen der Messfehler pro Ergebnis höher ist, sind bei deren Auswertung geringere Heritabilitätsschätzwerte zu erwarten. Weiterhin untersuchten BOICHARD et al. (1989) ausschließlich Tiere in der ersten Laktation. Auch die Anzahl untersuchter Herden und die verwandtschaftlichen Beziehungen zwischen den Herden sowie zwischen den Tieren einer Herde beeinflussen die Ergebnisse der genetischen Auswertung. BOICHARD et al. (1989) versuchten die verwandtschaftliche Verbindungen zwischen den untersuchten Herden durch die Berücksichtigung der Verwandtschaftsmatrix zwischen Vätern und die Hereinnahme der Nachkommen von in der künstlichen Besamung eingesetzten Böcken in die Auswer- 
tungen zu erhöhen. Weiterhin ist zu beachten, dass zwischen verschiedenen Rassen auch unterschiedlich hohe Heritabilitäten auftreten können. So fanden BÖMKES et al. (2003b) für die Weißen Deutschen Edelziegen mit einem Testtagstiermodell eine Heritabilität von $\mathrm{h}^{2}=0,30$ für die tägliche Milchmenge.

Die univariat und multivariat für die Fettmenge ermittelte Heritabilität von $\mathrm{h}^{2}=0,16$ stimmte mit den von BAGNICKA und LUKASZWEWICZ (1999) sowohl uni- als auch multivariat mit dem Tiermodell ermittelten Heritabilitäten gut überein. Die Schätzwerte für die Heritabilität der Eiweißmenge stimmten mit $\mathrm{h}^{2}=0,14$ bzw. $\mathrm{h}^{2}=$ 0,15 annähernd mit dem von BAGNICKA und LUKASZWEWICZ (1999) beschriebenen Wert von $\mathrm{h}^{2}=0,16$ überein. Allerdings beobachteten ILOEJE et al. (1981), KENNEDY et al. (1982a), BOICHARD et al. (1989) sowie BAGNICKA und LUKASZWEWICZ (1999) mit dem Vatermodell deutlich höhere Heritabilitätsschätzwerte sowohl für die Fett- als auch für die Eiweißmenge. Diese Unterschiede sind wiederum auf die unterschiedlichen Datenstrukturen, Auswertungsmethoden und möglicherweise auch auf Unterschiede zwischen Rassen zurückzuführen. Höhere Heritabilitätsschätzwerte ergaben sich auch in einem Tiermodell für die Fett- und Eiweißmenge bei französischen Ziegenrassen (BÉLICHON et al., 1995) und den Weißen Deutschen Edelziegen (BÖMKES et al., 2004b). Diese Unterschiede dürften vorwiegend auf rassebedingte Einflüsse zurückgehen, da die Weißen und Bunten Deutschen Edelziegen in den gleichen Regionen gehalten werden.

Die multivariat für den Fettgehalt ermittelte Heritabilität von $\mathrm{h}^{2}=0,17$ stimmte mit den von ANALLA et al. (1996) und BAGNICKA und LUKASZWEWICZ (1999) mit der gleichen Auswertungsmethode geschätzten Werten überein. Dagegen wurde univariat mit $h^{2}=0,27$ ein erheblich höherer Wert geschätzt, der jedoch weit unter den von ILOEJE et al. (1981), KENNEDY et al. (1982a), BOICHARD et al. (1989) sowie BAGNICKA und LUKASZWEWICZ (1999) mit dem Vatermodell geschätzten Werten blieb. Ebenso schätzten BÉLICHON et al. (1995) höhere Heritabilitäten für den Fettgehalt. Die in dieser Arbeit beobachteten Unterschiede zwischen der uni- und multivariaten Auswertung lassen sich durch einen Anstieg der Restvarianz erklären. Bei der Analyse der Weißen Deutschen Edelziegen konnte ein derartiger Effekt nicht beobachtet werden. Hier war die Heritabilität $\mathrm{mit}^{2}=0,25$ in der multivariaten Auswertung höher als bei der univariaten Analyse (BÖMKES et al., 2003b).

Für den Eiweißgehalt wurden mit $h^{2}=0,16$ bzw. $h^{2}=0,14$ deutlich geringere Werte als von ANALLA et al. (1996), BOICHARD et al. (1989), BÉLICHON et al. (1995) und BAGNICKA und LUKASZWEWICZ (1999) geschätzt, jedoch sehr ähnliche Werte wie für die Weißen Deutschen Edelziegen (BÖMKES et al., 2004b). Wie bereits erwähnt, werteten ANALLA et al. (1996), BOICHARD et al. (1989), BÉLICHON et al. (1995) und BAGNICKA und LUKASZWEWICZ (1999) jedoch Gesamtlaktationsleistungen und nicht Testtagsergebnisse aus, so dass geringere Werte zu erwarten waren.

Die Heritabilität des SCS wurde auf $h^{2}=0,10$ bzw. $h^{2}=0,16$ geschätzt und waren damit deutlich höher als für die Weißen Deutschen Edelziegen (BÖMKES et al., 2003b). Die geschätzten Werte stimmen mit denen von KENNEDY et al. (1982b), SEYKORA und MCDANIEL (1986), SCHUTZ et al. (1990), REENTS (1995) und CHRYSTAL et al. (1999) für Kühe beschriebenen Werten überein. Bei Ostfriesischen Milchschafen lagen die Heritabilitäten für den SCS mit $h^{2}=0,19$ höher als bei den Bunten Deutschen Edelziegen (HORSTICK et al., 2002). 
Zwischen der Milchmenge und der Fett- bzw. Eiweißmenge wurden für die Bunten Deutschen Edelziegen sehr hohe und positive additiv-genetische Korrelationen ermittelt. Zu gleichen Ergebnissen kamen BOICHARD et al. (1989), BAGNICKA und LUKASZWEWICZ (1999), KENNEDY et al. (1982a), BÉLICHON et al. (1995) und BÖMKES et al. (2003b). Die Milchmenge war im niedrigen bis mittleren Bereich und negativ mit dem Fett- bzw. Eiweißgehalt korreliert. Auch BOICHARD et al. (1989), ILOEJE et al. (1981), BÉLICHON et al. (1995) und BÖMKES et al. (2004b) kamen zu ähnlichen Ergebnissen. ANALLA et al. (1996) ermittelten ebenfalls negative, jedoch hohe additiv-genetische Korrelationen zwischen der Milchmenge und dem Fettbzw. Eiweißgehalt. Dagegen schätzten KENNEDY et al. (1982a) niedrige, aber positive additiv-genetische Korrelationen zwischen der Milchmenge und dem Fettgehalt von Alpinen, Saanen, Toggenburger und Nubischen Ziegen. In der Arbeit von BAGNICKA und LUKASZWEWICZ (1999) war der Fettgehalt niedrig, jedoch je nach verwendetem Modell negativ oder positiv mit der Milchmenge korreliert. Während die additiv-genetische Korrelation zwischen der Fettmenge und dem Fettgehalt für die Bunten wie auch die Weißen Deutschen Edelziegen auf $r_{g}=0,28$ geschätzt wurde, schätzten BOICHARD et al. (1989) und BÉLICHON et al. (1995) für Alpine und Saanenziegen höhere Werte im mittleren positiven Bereich. Zwischen der Eiweißmenge und dem Eiweißgehalt bestand mit $r_{g}=0,40$ eine additiv-genetische Korrelation im mittleren positiven Bereich. Dagegen schätzten BOICHARD et al. (1989) additiv-genetische Korrelationen von $r_{g}=0,29$ bzw. $r_{g}=0,14$. Bei den Weißen Deutschen Edelziegen war diese genetische Korrelation noch niedriger (BÖMKES et al., 2004b). Die additiv-genetische Korrelation zwischen dem Fett- und Eiweißgehalt lag in der Größenordnung der von BOICHARD et al. (1989) und BÉLICHON et al. (1995) für Alpine und Saanen Ziegen geschätzten Werten und stimmte mit dem Schätzergebnis für die Weißen Deutschen Edelziegen gut überein (BÖMKES et al., 2004b). Dagegen beobachteten ANALLA et al. (1996) sehr hohe positive additiv-genetische Korrelationen zwischen dem Fett- und Eiweißgehalt. Auch die von BAGNICKA und LUKASZWEWICZ (1999) mit verschiedenen Modellen zwischen dem Fettund Eiweißgehalt ermittelten additiv-genetischen Korrelationen lagen deutlich über den Schätzwerten für die Bunten und Weißen Deutschen Edelziegen. Während bisher in der Literatur zu den additiv-genetischen Korrelationen zwischen dem SCS und den Milchleistungsmerkmalen bei Milchziegen nur von BÖMKES et al. (2004b) berichtet wurde, liegen für Kühe sehr unterschiedliche Schätzergebnisse vor. So beschrieben KENNEDY et al. (1982b) niedrige und teilweise negative additiv-genetische Korrelationen zwischen diesen Merkmalen, wohingegen SEYKORA und MCDANIEL (1986) Korrelationen von annähernd Null für Kühe in der ersten Laktation fanden. SCHUTZ et al. (1990) ermittelten für Kühe in der ersten Laktation positive additiv-genetische Korrelationen, die in den späteren Laktationen geringer und zum Teil negativ wurden. In dieser Arbeit wurde hohe bis sehr hohe negative additiv-genetische Korrelationen zwischen dem SCS und der Milch-, Fett- und Eiweißmenge ermittelt. Im Gegensatz dazu bestanden niedrige, aber positive additiv-genetische Korrelationen zwischen dem SCS und Fett- sowie Eiweißgehalt. Dagegen waren die additiv-genetischen Korrelationen zwischen dem SCS und den Milchmengenmerkmalen bei den Weißen Deutschen Edelziegen im mittleren bis hohen Bereich positiv und die Gehaltsmerkmale negativ (BÖMKES et al., 2004b). Auf Grund der sehr unterschiedlichen Schätzwerte zwischen den Bunten und Weißen Deutschen Edelziegen sind sicherlich noch weitere Analysen 
mit umfangreicherem Material notwendig, um die hier gewonnenen Ergebnisse zu erhärten. Bei den Ostfriesischen Milchschafen lagen niedrige additiv-genetische Korrelationen zwischen den Milchleistungsmerkmalen und dem SCS vor. Nur zum Eiweißgehalt bestand eine nennenswerte additiv-genetische Korrelation von $r_{g}=0,14$ (Weiße Ostfriesische Milchschafe) bzw. $r_{g}=0,16$ (beide Farbrichtungen) (HORSTICK et al., 2002).

Die uni- und multivariat geschätzten Wiederholbarkeiten lagen mit Werten zwischen $\mathrm{w}=0,03$ und $\mathrm{w}=0,34$ erheblich unter den von BAGNICKA und LUKASZWEWICZ (1999), ANALLA et al. (1996) und ILOEJE et al. (1981) ermittelten Werten für verschiedene Milchleistungsmerkmale. Allerdings untersuchten diese Autoren Gesamtlaktationsleistungen von Ziegen, so dass sich die beschriebenen Wiederholbarkeiten auf wiederholt beobachtete Laktationsleistungen beziehen. Dagegen wurden in dieser Arbeit Testtagsergebnisse ausgewertet, bei denen es sich um wiederholte Leistungen innerhalb einer Laktation handelt. Die Wiederholbarkeit des SCS untersuchte keiner der zitierten Autoren. In dieser Arbeit ergab sich mit $\mathrm{w}=0,34$ bei der multivariaten Auswertung die insgesamt höchste ermittelte Wiederholbarkeit. Bei den Weißen Deutschen Edelziegen ergaben sich mit Ausnahme des SCS leicht höhere Schätzwerte für die Wiederholbarkeit (BÖMKES et al., 2004b). Insgesamt gesehen scheinen die Heritabilitäten und additiv-genetischen Varianzen der Milchmengenmerkmale und des Fettgehalts bei den Weißen Deutschen Edelziegen höher zu sein als bei den Bunten Deutschen Edelziegen, während für den Eiweißgehalt und SCS das Gegenteil zutrifft (BÖMKES et al., 2004b). Unterschiede infolge der Auswertungsmethode, der Datenstruktur oder Region sind weitgehend auszuschließen. Die negativen genetischen Korrelationen zwischen dem SCS und den Milchmengenmerkmalen sind züchterisch erwünscht, da eine Selektion auf Milchmenge zu keiner Beeinträchtigung der Eutergesundheit führen dürfte. Andererseits wird durch die gefundenen additiv-genetischen Korrelationen eine Selektion auf die Milchinhaltsstoffe Fett und Eiweiß erschwert, da deren additiv-genetischen Korrelationen zur Milchmenge negativ sind und gleichzeitig zum SCS positiv sind. Eine zu starke Betonung des Fett- und Eiweißgehalts der Milch in der Selektion würde zu einer verringerten Mengenleistung und zu höheren somatischen Zellgehalten und damit zu einer möglichen Verschlechterung der Eutergesundheit beitragen.

\section{Danksagung}

Der H. Wilhelm Schaumann Stiftung, Hamburg, sei für die Unterstützung dieser Arbeit sehr herzlich gedankt. Dem Ziegenzuchtverband Niedersachsen, dem Sächsischen Schaf- und Ziegenzuchtverband und dem Ziegenzuchtverband Baden-Württemberg danken wir sehr herzlich für die Bereitstellung der Daten.

\section{Literatur}

ANALLA, M.; JIMENEZ-GAMERO, I.; MUNOZ-SERRANO, A.; SERRADILLA, J.M.; FALAGAN, A.: Estimation of genetic parameters for milk yield and fat and protein contents of milk from MurcianoGranadina goats. J. Dairy Sci. 79 (1996), 1895-1898

BAGNICKA, E.; LUKASZEWICZ, M.: Genetic and environmental variation of dairy traits in a Polish population of goats. Animal Science Papers and Reports 17(1999), 59-65

BÉLICHON, S.; MANFREDI E.; PIACÈRE, A.:

Genetic parameters of dairy traits in the Alpine and Saanen goat breeds. Génét. Sél. Evol. 30 (1998), 529534 
BÖMKES, D.; HAMANN, H.; DISTL, O.:

Einfluss systematischer Umwelteffekte auf die Milchleistungsmerkmale bei Bunten Deutschen Edelziegen. Arch. Tierz., Dummerstorf (2004a), eingereicht

BÖMKES, D.; HAMANN, H.; DISTL, O.:

Populationsgenetische Analyse von Milchleistungsmerkmalen bei Weißen Deutschen Edelziegen. 2. Mitteilung: Genetische Parameter für Testtagsergebnisse. Züchtungskunde 76 (2004b), im Druck

BOICHARD, D.; BOULOG, N; RICORDEAU, G; PIACERE, A.; BARILLET; F.:

Genetic parameters for first lactation dairy traits in the Alpine and Saanen goat breeds. Génét. Sél. Evol. 21 (1989), 205-215

CHRYSTAL, M.A.; SEYKORA, A.J.; HANSEN, L.B.:

Heritabilities of teat end shape and teat diameter and their relationships with somatic cell score. J. Dairy

GALL, C.: Sci. 82 (1999), 2017-2022

Ziegenzucht. 2. Auflage, Ulmer Verlag, Stuttgart (2001), 50-54

HORSTICK, A.; HAMANN, H.; DISTL, O.:

Analyse von Milchleistungsmerkmalen bei ostfriesischen und schwarz-braunen Milchschafen. 2. Mitteilung: Genetische Parameter für Testtagsergebnisse. Züchtungskunde 73 (2002), 343-352

ILOEJE, M.U.; VAN VLECK, L.D.; WIGGANS, G.R.:

Components of variance for milk and fat yields in dairy goats. J. Dairy Sci. 64 (1981), 2290-2293

KENNEDY, B. W.; FINLEY, C.M.; BRADFORD, G.E.:

Phenotypic and genetic relationships between reproduction and milk production in dairy goats. J. Dairy Sci. 65 (1982a), 2373-2383

KENNEDY, B. W.; SETHAR, M.S.; MOXLEY, J.E.; DOWNEY, B.R.:

Heritability of somatic cell count and its relationship with milk yield and composition in Holsteins. J. Dairy Sci. 65 (1982b), 843-847

KOMINAKIS, A.; ROGDAKIS, E.; VASILOUDIS, CH.; LIASKOS, O.:

Genetic and environmental sources of variation of milk yield of Skopelos dairy goats. Small Rumin. Res. 36 (2000), 1-5

MAVROGENIS, A.P.; CONSTANTINOU, A.; LOUCA, A.:

Environmental and genetic causes of variation in production traits of Damascus goats. Anim. Prod. 38 (1984), 99-104

MAVROGENIS, A. P.; PAPACHRISTOFOROU, C.; LYSANDRIDES, P.; ROUSHIAS, A.:

Environmental and genetic effects on udder characteristics and milk production in Damascus goats. Small Rumin. Res. 2 (1989), 333-343

RÖNNINGEN, K.:

Causes of variation in the flavour intensity of goat milk. Acta. Agr. Scand. 15 (1965), 301

SCHUTZ, M.M.; HANSEN, L.B.; STEUERNAGEL, G.R.; RENEAU, J.K.; KUCK, A.L.:

Genetic parameters for somatic cells, protein, and fat in milk of Holsteins. J. Dairy Sci. 73 (1990), 494502

SEYKORA, A.J.; MCDANIEL, B.T.:

Genetics statistics and relationships of teat and udder traits, somatic cell counts, and milk production. J. Dairy Sci. 69 (1986), 2395-2407

Eingegangen: 08.10.2003

Akzeptiert: 27.02.2004

Anschrift der Verfasser

DOMINIKA BÖMKES, Dr. HENNING HAMANN, Prof. Dr. OTTMAR DISTL

Institut für Tierzucht und Vererbungsforschung der Tierärztliche Hochschule Hannover

Bünteweg 17p

D-30559 Hannover

E-Mail: ottmar.distl@tiho-hannover.de 